Philippe Jouan (Rouen)

\title{
OBSERVABILITY OF CONTROL SYSTEMS FOR POLYNOMIAL INPUTS AND GENERICITY
}

Abstract. We consider smooth single-input, two-output systems on a compact manifold $X$. We show that the set of systems that are observable for any polynomial input whose degree is less than or equal to a given bound contains an open and dense subset of the set of smooth systems.

1. Introduction. This paper deals with genericity of observability of control systems. Its purpose is to improve some well known results of J. P. Gauthier and I. Kupka. In [4], they have shown that, on a compact manifold $X$, smooth control systems $\Sigma=(h, f)$ :

$$
\left\{\begin{array}{l}
d x / d t=f(x, u), \quad u \in \mathbb{R}^{d_{u}}, y \in \mathbb{R}^{d_{y}}, \\
y=h(x, u),
\end{array}\right.
$$

are generically observable if the number of outputs is strictly larger than the number of inputs.

Here observability has the following meaning: a $k$-times differentiable input being given, the system is observable for this input if the mapping that associates to the initial state the outputs and their $2 d$ first derivatives at $t=0$ is an embedding: for this input the trajectories are instantaneously distinguished by the outputs.

This definition of observability is very strong but, in order to apply transversality theory to the genericity proofs, it is necessary to deal with a "differential" notion of observability. Moreover the above definition implies the weaker and more usual definitions of observability for one given input (see for example Definition 1), which turn out to require the universality of this input ([11]).

2000 Mathematics Subject Classification: 57N75, 57Q65, 93B07.

Key words and phrases: observability, genericity, transversality, polynomial input. 
To be more specific the results of [4] are the following: in the set of $C^{r}$-systems, and for $r$ sufficiently large:

1. For a given bound, the set of systems that are observable for every bounded and sufficiently differentiable input is dense.

2. For a given bound, the set of systems that are observable for every bounded and sufficiently differentiable input whose $2 d$ first derivatives are bounded contains an open and dense subset.

In the second statement, the derivatives of the input are assumed to be bounded. If this assumption is relaxed, the openness of the set of observable systems cannot be asserted and this is the point we want to improve in the present paper.

For the sake of clarity, we deal with the one-input, two-outputs case. But our results are genericity ones and therefore they are true in the one-input, $p$-outputs case for $p \geq 2$.

Moreover we deal with output mappings $h$ which depend on the input. This choice is of no importance and the results are the same in the case where the output is only a function of the state.

Our main result is Theorem 1:

Let $\sum^{r}$ be the set of $C^{r}$-systems on a compact d-dimensional manifold $X$ and let $s$ be an integer, $s \geq 2 d$. We consider bounded inputs with arbitrary $s$ first derivatives, but whose next derivatives up to order ds are not too large with respect to the $s$ first ones. The set of systems that are observable for every such input contains an open and dense subset of $\sum^{r}$.

To our knowledge this statement is at the moment the best genericity result in this topic; in particular we do not yet know if the set of systems observable for every bounded and sufficiently differentiable input contains an open and dense subset of $\sum^{r}$ or is only residual. The answer to this last question is of interest first for theoretical reasons, and second because some links between observability for $C^{\infty}$ (or $C^{\omega}$ ) inputs and continuous (or even $L^{\infty}$ ) inputs are known (see [9], [10], and [4] for the analytic case).

However Theorem 1 has the following corollary (Theorem 2):

Under the same assumptions the set of systems that are observable for every polynomial input whose value at $t=0$ is bounded by a fixed constant and whose degree is less than or equal to a given bound contains an open and dense subset of $\sum^{r}$.

The proof of Theorem 1 is based on a method of compactification of the inputs used for the first time in [8]. It was then applied to a particular case in $[2]$. 
1.1. Notations. $X$ is a smooth, compact, $d$-dimensional manifold; $\sum^{r}$ stands for the set of systems $\Sigma=(h, f)$ on $X$ where:

- $f$ is a $C^{r}$ vector field on $X$, parametrized by $u_{0} \in T, T$ being a 1-dimensional torus:

$$
f:\left(x, u_{0}\right) \in X \times T \mapsto f\left(x, u_{0}\right) \in T_{x} X .
$$

- $h$ is a $C^{r}$ mapping from $X \times T$ into $\mathbb{R}^{2}$.

Throughout the paper we will assume $r \in \mathbb{N} \cup\{\infty, \omega\}$ to be large enough to give sense to the statements and computations considered. If $r \in \mathbb{N} \cup\{\infty\}$ the set $\sum^{r}$ is endowed with the $C^{r}$-topology (without ambiguity since the set $X \times T$ is compact (cf. [7])). In the real-analytic case the topology is induced on $\sum^{\omega}$ by the $C^{\infty}$-topology.

$f^{k}$ stands for the $k$-times extended vector field, that is, the vector field on $X \times T \times \mathbb{R}^{k-1}$ controlled by $u_{k}$ and defined by

$$
f^{k}\left(x, u_{0}, u_{1}, \ldots, u_{k}\right)=f\left(x, u_{0}\right)+\sum_{i=0}^{k-1} u_{i+1} \frac{\partial}{\partial u_{i}} .
$$

We will denote by $u$ the $k$-uple $\left(u_{1}, \ldots, u_{k}\right)$ without any reference to the integer $k$ when this is not confusing.

A system $\Sigma=(h, f)$ with $h=\left(h_{1}, h_{2}\right)$ being given, we consider the following mapping:

$$
\left(x, u_{0}, u\right) \mapsto\left(\begin{array}{c}
R_{k, \Sigma}: X \times T \times \mathbb{R}^{k-1} \rightarrow \mathbb{R}^{2 k}, \\
h_{1}\left(x, u_{0}\right), h_{2}\left(x, u_{0}\right), \\
L_{f^{k}} h_{1}\left(x, u_{0}, u\right), L_{f^{k}} h_{2}\left(x, u_{0}, u\right), \\
\ldots \\
L_{f^{k}}^{k-1} h_{1}\left(x, u_{0}, u\right), L_{f^{k}}^{k-1} h_{2}\left(x, u_{0}, u\right)
\end{array}\right) .
$$

Clearly if an input $\mathbf{u}(t)$ such that $\frac{d^{i}}{d t^{i}}(\mathbf{u}(0))=u_{i}$ for $i=0, \ldots, k-1$ is applied to $\Sigma$, then $R_{k, \Sigma}\left(\cdot, u_{0}, u\right)$ is the mapping that, to an initial state $x_{0} \in X$, associates the output and its first $k-1$ derivatives at $t=0$. If $U$ is a subset of $\mathbb{R}^{k-1}$, the injectivity of $R_{k, \Sigma}\left(\cdot, u_{0}, u\right)$ for every $\left(u_{0}, u\right) \in T \times U$ implies the observability of $\Sigma$ for every $C^{k-1}$-input $t \mapsto \mathbf{u}(t)$ such that $\left(\frac{d}{d t}(\mathbf{u}(t)), \frac{d^{2}}{d t^{2}}(\mathbf{u}(t)), \ldots, \frac{d^{k-1}}{d t^{k-1}}(\mathbf{u}(t))\right) \in U$.

Finally let us define the "suspension map" $S R_{k, \Sigma}$ by

$$
\begin{gathered}
S R_{k, \Sigma}: X \times T \times \mathbb{R}^{k-1} \rightarrow \mathbb{R}^{2 k} \times T \times \mathbb{R}^{k-1}, \\
S R_{k, \Sigma}\left(x, u_{0}, u\right)=\left(R_{k, \Sigma}\left(x, u_{0}, u\right), u_{0}, u\right) .
\end{gathered}
$$




\subsection{Summary of the previous results}

1. Let $B>0$ and $I_{B}=[-B, B]$. The set of systems $\Sigma$ such that the restriction of $S R_{2 d+1, \Sigma}$ to $X \times T \times\left(I_{B}\right)^{2 d}$ is an embedding is open and dense in $\sum^{r}$ (Theorem II.3 of [4]).

2. The set of systems $\Sigma$ such that $S R_{2 d+1, \Sigma}$ is an embedding is residual, hence dense in $\sum^{r}$ (Theorem II.2 of [4]).

3 . The set of systems $\Sigma$ such that $S R_{2 d+1, \Sigma}$ is an immersion (not necessarily injective) contains an open and dense subset of $\sum^{r}$ (Theorem II.1 of $[4])$.

4. The set of systems $\Sigma$ such that $S R_{2 d+1, \Sigma}(\cdot, \cdot, u)$ is one-to-one "far from the diagonal" for any $u \in \mathbb{R}^{k-1}$ is open and dense in $\sum^{r}$; here "far from the diagonal" has the following meaning: for every compact subset $K$ of $X \times X \backslash \Delta X$, where $\Delta X$ is the diagonal of $X \times X$, the set of systems such that

$$
\left(x_{1}, x_{2}, u_{0}, u\right) \in K \times T \times \mathbb{R}^{2 d} \mapsto R_{k, \Sigma}\left(x_{1}, u_{0}, u\right)-R_{k, \Sigma}\left(x_{2}, u_{0}, u\right)
$$

does not vanish is open and dense in $\sum^{r}$. This last result is not explicitly stated in [4]. It is however stated and proved at the end of Section III.1 of $[3]$.

2. Input compactification and statement of the results. Let $\Sigma=$ $(h, f) \in \sum^{r}$ and $\lambda \in \mathbb{R}_{+}^{*}$. Then $\Sigma_{\lambda}$ stands for the system $(h, \lambda f)$, and for the same positive number $\lambda$ (and for any $k \geq 1$ ):

- $\Delta_{\lambda}$ is the mapping from $\mathbb{R}^{k}$ into $\mathbb{R}^{k}$ defined by

$$
\Delta_{\lambda} u=\left(\lambda u_{1}, \lambda^{2} u_{2}, \ldots, \lambda^{k} u_{k}\right) .
$$

- $A_{\lambda}$ is the mapping from $\mathbb{R}^{2 k}$ into $\mathbb{R}^{2 k}$ defined by

$$
A_{\lambda}\left(y_{1}, z_{1} ; y_{2}, z_{2} ; \ldots ; y_{k}, z_{k}\right)=\left(y_{1}, z_{1} ; \lambda y_{2}, \lambda z_{2} ; \ldots ; \lambda^{k-1} y_{k}, \lambda^{k-1} z_{k}\right) .
$$

Lemma 1. For every system $\Sigma \in \sum^{r}, \lambda \in \mathbb{R}_{+}^{*}$ and $k \geq 1$, we have

$$
R_{k, \Sigma_{\lambda}}\left(\cdot, \cdot, \Delta_{\lambda} u\right)=A_{\lambda} R_{k, \Sigma}(\cdot, \cdot, u) .
$$

Proof. Let $u=\left(u_{1}, \ldots, u_{k-1}\right) \in \mathbb{R}^{k-1}$ and let $t \mapsto \mathbf{u}(t)$ be a $(k-1)$-times differentiable input such that

$$
\frac{d^{i} \mathbf{u}}{d t^{i}}(0)=u_{i} \quad \text { for } i=1, \ldots, k-1 .
$$

Denote by $t \mapsto \phi(t)$ the solution of $\dot{x}=f\left(x, u_{0}\right)$ for the initial condition $\phi(0)=x$ for the input $\mathbf{u}(t)$. The integral curve $\phi$ satisfies

$$
\frac{d}{d t}(\phi(\lambda t))=\lambda f(\phi(\lambda t), \mathbf{u}(\lambda t))
$$


and therefore $t \mapsto \phi(\lambda t)$ is the solution of $\dot{x}=\lambda f\left(x, u_{0}\right)$ for the initial condition $x$ and the input $t \mapsto \mathbf{u}(\lambda t)$. Now Lemma 1 is a consequence of

and

$$
\left.\frac{d^{i}}{d t^{i}}(h \circ \phi(\lambda t))\right|_{t=0}=\lambda^{i} \frac{d^{i}}{d t^{i}}(h \circ \phi)(0) \quad \text { for } i=1, \ldots, k-1
$$

$$
R_{k, \Sigma}(x, \mathbf{u}(0), u)=\left(h(\phi(t)), \frac{d}{d t}(h(\phi(t))), \ldots, \frac{d^{k-1}}{d t^{k-1}}(h(\phi(t)))\right)_{t=0} .
$$

The above lemma allows us to replace the study of the injectivity of $R_{k, \Sigma}\left(\cdot, u_{0}, u\right)$ for every $\left(u_{0}, u\right) \in T \times \mathbb{R}^{k-1}$ by the study of the injectivity of $R_{k, \Sigma_{\lambda}}\left(\cdot, u_{0}, u\right)$ for every $\left(u_{0}, u\right) \in T \times[-1,1]^{k-1}$ and every $\lambda>0$.

Let $s \geq 2 d$ be an integer. For $u=\left(u_{1}, \ldots, u_{s}\right) \in \mathbb{R}^{s}$, let

$$
\lambda(u)=\max _{1 \leq i \leq s}\left|u_{i}\right|^{1 / i} .
$$

If $u \neq 0$ we can define $v_{i}=u_{i} /(\lambda(u))^{i}$ for $i=1, \ldots, s$. Obviously $\left|v_{i}\right|^{1 / i}=$ $\left|u_{i}\right|^{1 / i} / \lambda(u) \leq 1$ and, if $i_{0}$ yields the maximum, then $\left|v_{i_{0}}\right|^{1 / i_{0}}=1$.

Let $v=\left(v_{1}, \ldots, v_{s}\right) \in \mathbb{R}^{s}$. We have

Finally, set

$$
v=\Delta_{\lambda(u)^{-1}}(u) \text { and }\|v\|_{\infty}=1 \text {. }
$$

$$
C_{s}=\left\{w \in \mathbb{R}^{s} ; \max _{1 \leq i \leq s}\left|w_{i}\right|=1\right\}
$$

Then $C_{s}$ is compact and $u \neq 0 \Rightarrow v \in C_{s}$.

We denote by $G_{s}$ the set of elements $(u, \bar{u})=\left(u_{1}, \ldots, u_{s} ; u_{s+1}, \ldots, u_{d s}\right)$ in $\mathbb{R}^{d s}$ (recall that $d=\operatorname{dim} X$ ) that satisfy at least one of the conditions:

(i) $\max _{1 \leq i \leq s}\left|u_{i}\right| \leq 1$,

(ii) $\left|u_{k}\right|^{1 / k} \leq \lambda(u)$ for $s+1 \leq k \leq d s$,

We can now state the main result of this paper:

THEOREM 1. The set of systems $\Sigma \in \sum^{r}$ such that the restriction of $S R_{d s+1, \Sigma}$ to $X \times T \times G_{s}$ is an embedding contains an open and dense subset of $\sum^{r}($ for $r \in \mathbb{N} \cup\{\infty, \omega\}$ large enough $)$.

Before giving the proof, we apply Theorem 1 to observability for polynomial inputs. We first recall the usual definition of observability: an input being given, we require that any two different initial states are instantaneously distinguished by the outputs. More specifically:

DeFinition 1. The system $\Sigma \in \sum^{r}$ is said to be observable for an input $u \in L^{\infty}\left[0, T_{u}\right)$ if

$$
\begin{gathered}
\forall\left(x_{1}, x_{2}\right) \in X \times X, x_{1} \neq x_{2}, \quad \forall T>0 \\
\exists \tau \in[0, T) \quad \text { such that } h\left(x_{1}(\tau)\right) \neq h\left(x_{2}(\tau)\right)
\end{gathered}
$$

where $x_{i}(\tau)$ is the trajectory of $\Sigma$ starting from $x_{i}$. 
We can now state:

THEOREM 2. Let $s$ be a positive integer and let $B>0$. The set of systems $\Sigma \in \sum^{r}$ that are observable for every polynomial input $\mathbf{u}$ with $|\mathbf{u}(0)| \leq B$ and whose degree is less than or equal to $s$ contains an open and dense subset of $\sum^{r}($ for $r \in \mathbb{N} \cup\{\infty, \omega\}$ large enough $)$.

3. Proof of Theorem 1. The proof makes use of the following lemma:

LemMa 2. For $h \in C^{r}\left(X \times T ; \mathbb{R}^{2}\right), r$ large enough, define the mapping

$$
\Phi(h)=\left(h, \frac{\partial h}{\partial u_{0}}, \ldots, \frac{\partial^{d} h}{\partial u_{0}^{d}}\right) .
$$

The set $\mathcal{O}$ of functions $h \in C^{r}\left(X \times T ; \mathbb{R}^{2}\right)$ such that the rank of $d_{x} \Phi(h)$ is $d=\operatorname{dim} X$ for every $\left(x, u_{0}\right) \in X \times T$ is open and dense in $C^{r}\left(X \times T ; \mathbb{R}^{2}\right)$.

Proof. Let

$$
Y=\bigcup_{\left(x, u_{0}\right) \in X \times T}\left(T_{x}^{*} X\right)^{(2 d+2)} .
$$

Then $Y$ is a vector bundle over $X \times T$. Let $W$ be the set of elements $\left(v_{1}, \ldots, v_{2 d+2}\right)$ of $Y$ whose extracted $d$-uples $\left(v_{i_{1}}, \ldots, v_{i_{d}}\right)$ are all linearly dependent. Then $W$ is closed in $Y$ and is a finite union of submanifolds of $Y$. Its codimension (i.e. the codimension of its biggest stratum) is equal, by the product of coranks theorem, to $(d-(d-1))(2 d+2-(d-1))=d+3$ (cf. [5]).

Let $\varrho$ be the mapping from $C^{r}\left(X \times T ; \mathbb{R}^{2}\right)$ into $C^{r-d-1}(X \times T ; Y)$ defined by

$$
\varrho(h)=d_{x} \Phi(h) .
$$

The following equivalences hold, where $帀$ means "is transversal to":

$$
h \in \mathcal{O} \Leftrightarrow \varrho(h)(X \times T) \cap W=\emptyset \Leftrightarrow \varrho(h) \mp W
$$

because codim $W>\operatorname{dim} X \times T$.

Let $\mathrm{ev}_{\varrho}: C^{r}\left(X \times T ; \mathbb{R}^{2}\right) \times X \times T \rightarrow Y$ be the evaluation map defined by

$$
\operatorname{ev}_{\varrho}\left(h, x, u_{0}\right)=\varrho(h)\left(x, u_{0}\right) .
$$

Then $\mathrm{ev}_{\varrho}$ is of class $C^{1}$ if $r$ is large enough. Let us prove that

$$
\forall\left(h^{0}, x^{0}, u_{0}^{0}\right) \in C^{r}\left(X \times T ; \mathbb{R}^{2}\right) \times X \times T \quad \mathrm{ev}_{\varrho} \pitchfork_{\left(h^{0}, x^{0}, u_{0}^{0}\right)} W .
$$

As the mapping $\mathrm{ev}_{\varrho}$ is linear and continuous with respect to its first variable, it is sufficient to show that the mapping

$$
h \mapsto T_{h^{0}} \mathrm{ev}_{\varrho}\left(\cdot, x^{0}, u_{0}^{0}\right) \cdot h=\varrho(h)\left(x^{0}, u_{0}^{0}\right)
$$


is surjective for every $\left(x^{0}, u_{0}^{0}\right) \in X \times T$. Let $\left(x_{1}, \ldots, x_{d} ; w\right)$ be local coordinates at $\left(x^{0}, u_{0}^{0}\right)$ and let $v \in\left(T_{x^{0}}^{*} X\right)^{(2 d+2)}$. In these coordinates,

$$
v=\left(v_{0}^{1}, v_{0}^{2}, v_{1}^{1}, v_{1}^{2}, \ldots, v_{d}^{1}, v_{d}^{2}\right)
$$

where

$$
v_{j}^{i}=\sum_{k=1}^{d} v_{j, k}^{i} d x_{k}, \quad i=1,2, j=0, \ldots, d .
$$

Let $h=\left(h^{1}, h^{2}\right)$ be such that locally

$$
h^{i}=\sum_{j=0}^{d}\left(\sum_{k=1}^{d} v_{j, k}^{i} x_{k}\right) \frac{w^{j}}{j !} .
$$

Obviously $\varrho(h)\left(x^{0}, u^{0}\right)=v$ and the required surjectivity is proved.

Thus the mapping ev $\varrho$ is transversal to $W$ and, by Theorems 18.2 and 19.1 of Abraham-Robbin [1], the set of functions $h \in C^{r}\left(X \times T ; \mathbb{R}^{2}\right)$ such that $\varrho(h)$ is transversal to $W$, that is, the set $\mathcal{O}$, is open and dense in $C^{r}\left(X \times T ; \mathbb{R}^{2}\right)$.

REMARK. The transversality theorems of Abraham-Robbin are stated for $r<\infty$. However Lemma 2 remains true in the $C^{\infty}$ case because the $C^{\infty}$ topology is the inductive limit of the $C^{r}$-topologies. Following the method proposed in [4] it can be shown that Lemma 2 is also true in the $C^{\omega}$ case.

Proof of Theorem 1. Denote by $\Re$ the set of systems $\Sigma=(h, f)$ such that:

1. $h$ belongs to the set $\mathcal{O}$ defined in Lemma 2 .

2. $S R_{2 d+1, \Sigma}$ is an embedding.

3. $\Sigma$ belongs to the interior of the set of systems $\Sigma^{\prime}$ for which $S R_{2 d+1, \Sigma^{\prime}}$ is an immersion.

The set $\Re$ is residual in $\sum^{r}$. Fix $\Sigma=(h, f) \in \Re$. There exists a neighbourhood $V$ of $\Sigma$ in $\sum^{r}$ all of whose elements $\Sigma^{\prime}$ are such that $S R_{2 d+1, \Sigma^{\prime}}$ is an immersion. As $X$ is compact, $S R_{2 d+1, \Sigma^{\prime}}$ is in fact a proper immersion. Hence, in order to prove Theorem 1, it is sufficient to show that for every sequence $\left(\Sigma^{n}\right)_{n \geq 1}$ of systems that tends to $\Sigma$ in $\sum^{r}$, the restrictions of the mappings $S R_{d s+1, \Sigma^{n}}$ to $X \times T \times G_{s}$ are one-to-one for $n$ large enough. So let $\left(\Sigma^{n}\right)_{n \geq 1}$ be such a sequence and assume that for each $n$, one can find $x_{1}^{n} \neq x_{2}^{n} \in X, u_{0}^{n} \in T,\left(u^{n}, \bar{u}^{n}\right) \in G_{s}$ such that

$$
R_{d s+1, \Sigma^{n}}\left(x_{1}^{n}, u_{0}^{n},\left(u^{n}, \bar{u}^{n}\right)\right)=R_{d s+1, \Sigma^{n}}\left(x_{2}^{n}, u_{0}^{n},\left(u^{n}, \bar{u}^{n}\right)\right) .
$$

$X$ being compact, we can extract a subsequence such that

$$
x_{1}^{n} \rightarrow x_{1}, \quad x_{2}^{n} \rightarrow x_{2}
$$

as $n \rightarrow \infty$. Then $x_{1}=x_{2}$. Indeed, $\left(x_{1}^{n}, x_{2}^{n}\right)$ tends to $\left(x_{1}, x_{2}\right)$ in $X \times X$. 
If $\left(K_{p}\right)_{p \geq 1}$ is an increasing sequence of compact subsets of $X \times X \backslash \Delta X$ such that $\bigcup_{p \geq 1} K_{p}=X \times X \backslash \Delta X$, then result 4 recalled in Section 1.2 implies that $\left(x_{1}, x_{2}\right)$ belongs to none of the sets $K_{p}$. Hence $\left(x_{1}, x_{2}\right) \in \Delta X$.

Let $x=x_{1}=x_{2}$ and choose a coordinate neighbourhood of $x$. We can assume that $x_{1}^{n}$ and $x_{2}^{n}$ belong to this neighbourhood for every $n$. Thus we can define

$$
b_{n}=\frac{1}{\left\|x_{2}^{n}-x_{1}^{n}\right\|}\left(x_{2}^{n}-x_{1}^{n}\right), \quad n \geq 1,
$$

where $\|\cdot\|$ stands for the Euclidean norm in $\mathbb{R}^{d}$; notice that $b_{n}$ belongs to the sphere $S^{d-1}$.

Furthermore, result 1 of Section 1.2 implies

$$
\left\|u^{n}\right\|_{\infty} \rightarrow \infty
$$

Set $\lambda_{n}=\lambda\left(u^{n}\right)$ as in Section 2. We have $\lambda_{n} \rightarrow \infty$ and we can assume $\lambda_{n} \neq 0$ for every $n$. Thus it is possible to define $\left(v^{n}, \bar{v}^{n}\right)=\Delta_{\lambda_{n}^{-1}}\left(u^{n}, \bar{u}^{n}\right)$. Then $v^{n} \in C_{s}$ and $\left\|\bar{v}^{n}\right\|_{\infty} \leq 1$ for every $n$.

Finally, extracting a subsequence again if necessary, we have the following convergences as $n \rightarrow \infty$ :

$$
\begin{array}{cl}
\left(x_{1}^{n}, x_{2}^{n}\right) \rightarrow(x, x) \in \Delta X, & b_{n} \rightarrow b \in S^{d-1}, \quad u_{0}^{n} \rightarrow u_{0} \in T, \\
v^{n} \rightarrow v \in C_{s}, & \bar{v}^{n} \rightarrow \bar{v}, \quad\|\bar{v}\|_{\infty} \leq 1 .
\end{array}
$$

In order to simplify the forthcoming computations we let

$$
\delta^{n}=R_{d s+1, \Sigma_{\lambda_{n}^{-1}}^{n}}\left(x_{2}^{n}, u_{0}^{n},\left(v^{n}, \bar{v}^{n}\right)\right)-R_{d s+1, \Sigma_{\lambda_{n}^{-1}}^{n}}\left(x_{1}^{n}, u_{0}^{n},\left(v^{n}, \bar{v}^{n}\right)\right) .
$$

According to Lemma 1,

$$
\begin{aligned}
\delta^{n} & =A_{\lambda_{n}^{-1}}\left(R_{d s+1, \Sigma^{n}}\left(x_{2}^{n}, u_{0}^{n},\left(u^{n}, \bar{u}^{n}\right)\right)-R_{d s+1, \Sigma^{n}}\left(x_{1}^{n}, u_{0}^{n},\left(u^{n}, \bar{u}^{n}\right)\right)\right) \\
& =0 \quad \text { by assumption. }
\end{aligned}
$$

Again to simplify the forthcoming computations we let

$$
h^{(l)}=d_{x} \frac{\partial^{l} h}{\partial u_{0}^{l}}\left(x, u_{0}\right) \cdot b .
$$

From now on $v_{i}^{j}$ will stand for $\left(v_{i}\right)^{j}$, and not for the $i$ th coordinate of $\left(v^{j}, \bar{v}^{j}\right)$. We have

$$
\lim _{n \rightarrow \infty} \frac{1}{\left\|x_{2}^{n}-x_{1}^{n}\right\|} \delta^{n}=\left(L_{0}, L_{1}, \ldots, L_{d s}\right)
$$

where

$$
\begin{aligned}
& L_{0}=h^{(0)} \\
& L_{1}=v_{1} h^{(1)} \\
& L_{2}=v_{1}^{2} h^{(2)}+v_{2} h^{(1)}, \ldots, \\
& L_{k}=P_{k, k} h^{(k)}+\ldots+P_{k, 1} h^{(1)}, \ldots,
\end{aligned}
$$


where $P_{k, l}$ is a polynomial in $v_{1}, \ldots, v_{k}$, and $L_{k} \in \mathbb{R}^{2}$ for $0 \leq k \leq d s$. Indeed, denote by $\delta_{k}^{n}, 0 \leq k \leq d s$, the $k$ th double-coordinate of $\delta^{n}$. Then $\delta_{k}^{n}$ is a polynomial in the $k$ first coordinates of $\left(v^{n}, \bar{v}^{n}\right)$ whose coefficients are the derivatives of $h$ with respect to the vector field $\lambda_{n}^{-1} f$ and with respect to $\partial / \partial u_{0}$ of total order less than or equal to $k$. Since $\lambda_{n}^{-1} \rightarrow 0$, and since all the other variables belong to compact sets, all the monomials where a derivation with respect to $\lambda_{n}^{-1} f$ occurs vanish as $n$ tends to infinity.

Moreover, for $1 \leq k \leq d s, P_{k, l}$ is a sum of monomials of the kind

$$
\zeta v_{1}^{p_{1}} v_{2}^{p_{2}} \ldots v_{k}^{p_{k}}
$$

where $\zeta \in \mathbb{N}^{*}$ and

$$
\left\{\begin{array}{l}
p_{1}+p_{2}+\ldots+p_{k}=l \\
p_{1}+2 p_{2}+\ldots+k p_{k}=k
\end{array} .\right.
$$

We prove $(*)$ by induction. Clearly $L_{1}=v_{1} h^{(1)}$ and $(*)$ is true for $k=1$. Assume it is true for $L_{k}$. Now, $L_{k+1}$ is obtained by differentiation of $L_{k}$ with respect to $\partial u_{0}=v_{1} \partial / \partial u_{0}$ and $\partial v_{i}=v_{i+1} \partial / \partial v_{i}$ for $1 \leq i \leq k$. Let

$$
L_{k}=P_{k, k} h^{(k)}+\ldots+P_{k, 1} h^{(1)}
$$

and let $m=\zeta v_{1}^{p_{1}} v_{2}^{p_{2}} \ldots v_{k}^{p_{k}}$ be a monomial of $P_{k, l}$ where $1 \leq l \leq k$. By hypothesis we have

$$
\left\{\begin{array}{l}
p_{1}+p_{2}+\ldots+p_{k}=l \\
p_{1}+2 p_{2}+\ldots+k p_{k}=k
\end{array}\right.
$$

- If $m h^{(l)}$ is differentiated with respect to $\partial u_{0}$, then

$$
\partial u_{0}\left(m h^{(l)}\right)=v_{1} m h^{(l+1)}
$$

and $v_{1} m=\zeta v_{1}^{p_{1}+1} v_{2}^{p_{2}} \ldots v_{k}^{p_{k}}$ is a monomial of $P_{k+1, l+1}$ which satisfies

$$
\left\{\begin{array}{l}
\left(p_{1}+1\right)+p_{2}+\ldots+p_{k}=l+1 \\
\left(p_{1}+1\right)+2 p_{2}+\ldots+k p_{k}=k+1
\end{array}\right.
$$

- If $m h^{(l)}$ is differentiated with respect to $\partial v_{i}, 1 \leq i \leq k$, then, assuming $p_{i} \geq 1$, we have

$$
\partial v_{i}\left(m h^{(l)}\right)=p_{i} \zeta v_{1}^{p_{1}} v_{2}^{p_{2}} \ldots v_{i}^{p_{i}-1} v_{i+1}^{p_{i+1}+1} \ldots v_{k}^{p_{k}} h^{(l)}
$$

and $p_{i} \zeta v_{1}^{p_{1}} v_{2}^{p_{2}} \ldots v_{i}^{p_{i}-1} v_{i+1}^{p_{i+1}+1} \ldots v_{k}^{p_{k}} h^{(l)}$ is a monomial of $P_{k+1, l}$ which satisfies

$$
\left\{\begin{array}{l}
p_{1}+p_{2}+\ldots+\left(p_{i}-1\right)+\left(p_{i+1}+1\right)+\ldots+p_{k}=l \\
p_{1}+2 p_{2}+\ldots+i\left(p_{i}-1\right)+(i+1)\left(p_{i+1}+1\right)+\ldots+k p_{k} \\
\quad=k-i+(i+1)=k+1
\end{array}\right.
$$

Therefore property $(*)$ is true for $L_{k+1}$. 
A consequence is the existence of an integer $j$, with $1 \leq j \leq k / l$, for which $p_{j} \neq 0$. Otherwise we would have

$$
\sum_{1 \leq i \leq k} i p_{i}>\frac{k}{l} l=k
$$

Since $\delta^{n}$ vanishes for every $n$, the equalities $L_{0}=L_{1}=\ldots=L_{d s}=0$ must hold. Let us show that this is impossible:

Let $r, 0 \leq r \leq d$, be the smallest integer for which

$$
h^{(r)}=d_{x} \frac{\partial^{r} h}{\partial u_{0}^{r}}\left(x, u_{0}\right) \cdot b \neq 0 .
$$

The integer $r$ exists by Lemma 2. If $r=0$, then $L_{0}=h^{(0)} \neq 0$. Otherwise let $q, 1 \leq q \leq s$, be the smallest integer for which $v_{q} \neq 0$. Such a $q$ exists because $v \in C_{s}$. Moreover we have

$$
r q \leq d s
$$

It remains to show that $L_{r q} \neq 0$. We have

$$
L_{r q}=\sum_{l=1}^{r q} P_{r q, l} h^{(l)}
$$

- If $l<r$, then $h^{(l)}=0$ by assumption and $P_{r q, l} h^{(l)}=0$.

- If $l>r$, consider a monomial $m$ of $P_{r q, l}$ :

$$
m=\zeta v_{1}^{p_{1}} v_{2}^{p_{2}} \ldots v_{r q}^{p_{r q}} .
$$

There exists an integer $i \leq k / l=r q / l<q$ for which $p_{i} \neq 0$. But $i<q$ implies $v_{i}=0$ and the monomial $m$ vanishes. Hence $P_{r q, l}=0$.

- There remains the case $l=r$. A monomial of $P_{r q, r}$ is $\zeta v_{q}^{r}, \zeta \neq 0$. It is straightforward to verify that $\zeta v_{q}^{r} h^{(r)}$ can be obtained by a suitable differentiation of $L_{0}=h^{(0)}$.

Let $\zeta v_{1}^{p_{1}} v_{2}^{p_{2}} \ldots v_{r q}^{p_{r q}}$ be another monomial of $P_{r q, r}$. There exists at least one integer $j \neq r$ for which $p_{j} \neq 0$, and

$$
\begin{gathered}
p_{1}+p_{2}+\ldots+p_{q}+\ldots+p_{p q}=r \\
p_{1}+2 p_{2}+\ldots+q p_{q}+\ldots+r q p_{r q}=r q .
\end{gathered}
$$

Hence

$$
\frac{1}{q} p_{1}+\frac{2}{q} p_{2}+\ldots+p_{q}+\ldots+r p_{p q}=p_{1}+p_{2}+\ldots+p_{q}+\ldots+p_{r q} .
$$

If $p_{1}=p_{2}=\ldots=p_{q-1}=0$, then

$$
\frac{q+1}{q} p_{q+1}+\frac{q+2}{q} p_{q+2}+\ldots+r p_{r q}=p_{q+1}+p_{q+2}+\ldots+p_{r q} \text {. }
$$


But this is impossible because $p_{j} \neq 0$ for at least one integer $j>q$. Therefore at least one of the integers $p_{j}, 1 \leq j \leq q-1$, does not vanish and, since $v_{j}=0$, the monomial vanishes.

Finally the only monomial of $L_{r q}$ that does not vanish is $\zeta v_{q}^{r} h^{(r)}$ :

$$
L_{r q}=\zeta v_{q}^{r} h^{(r)} \neq 0 .
$$

This is a contradiction and Theorem 1 is proved.

\section{References}

[1] R. Abraham and J. Robbin, Transversal Mappings and Flows, Benjamin, 1967.

[2] M. Balde and P. Jouan, Genericity of observability of control-affine systems, Control Optim. Calc. Var. 3 (1998), 345-359.

[3] J.-P. Gauthier and I. Kupka, Genericity of observability and the existence of nonlinear observers, in: Geometry in Nonlinear Control and Differential Inclusions, Banach Center Publ. 32, Inst. Math., Polish Acad. Sci., 1995, 227-244.

[4] - - - Observability for systems with more outputs than inputs and asymptotic observers, Math. Z. 223 (1996), 47-78.

[5] M. Golubitsky and V. Guillemin, Stable Mappings and their Singularities, Grad. Texts in Math. 14, Springer, 1973.

[6] R. Hermann and A. J. Krener, Nonlinear controllability and observability, IEEE Trans. Automat. Control AC-22 (1977), 728-740.

[7] M. W. Hirsch, Differential Topology, Grad. Texts in Math. 33, Springer, 1976.

[8] P. Jouan, Singularités des systèmes non linéaires. Observabilité et observateurs, thèse de l'Université de Rouen, 1995.

[9],$- C^{\infty}$ and $L^{\infty}$ observability of single-input $C^{\infty}$-systems, J. Dynam. Control Systems 7 (2001), 151-169.

[10] -, Observability of $C^{\infty}$-systems for $L^{\infty}$-single-inputs and some examples, in: Nonlinear Control in the Year 2000, Vol. 1, Springer, 2001, 575-582.

[11] H. J. Sussmann, Single-input observability of continuous-time systems, Math. Systems Theory 12 (1979), 371-393.

Laboratoire R. Salem, UMR CNRS 6085

Université de Rouen, Mathématiques

Site Colbert

76821 Mont-Saint-Aignan Cedex, France

E-mail: Philippe.Jouan@univ-rouen.fr

Received on 31.7.2000;

revised version on 26.3.2001 